\title{
ASSESSMENT OF PHYSICO-CHEMICAL QUALITY OF BORE WELL WATER SAMPLES OF SAGAR CITY, MP, INDIA
}

\author{
Hemant PATHAK* \\ Department of Chemistry, Indira Gandhi Govt. Engineering College, Sagar, (M.P.), India, \\ e-mail: hemantp1981@yahoo.co.in
}

\begin{abstract}
Citation: Pathak, H. (2020). Assessment of Physico-Chemical Quality of Bore Well Water Samples of Sagar City, MP, India. Analele Universităţii din Oradea, Seria Geografie, 30(1), 48-52. https://doi.org/10.30892/auog.301106-826

Abstract: Bore well water is one of the major resources of the drinking water in Sagar city (M.P.). In the present study samples collected from different localities in Sagar (MP) were analyzed for their physico-chemical characteristics were carried out during different months of the pre monsoon, monsoon and post monsoon seasons in July 2018 to Aug 2019 Results shows that all the samples are under Indian standard limit for drinking purpose. The statistical analysis of the collected samples yielded the Matrix of Pearson Correlation. On the basis of analyses parameters, the results indicated the, satisfactory water quality of the Bore well samples.
\end{abstract}

Key words: Physico-chemical quality, Bore well water

$* \quad * \quad * \quad * \quad * *$

\section{INTRODUCTION}

Water is the elixir for life. Adequate supply of potable safe water is absolutely essential and is the basic need for all human being on the earth (Romocea et al., 2018; Herman et al., $2019 \mathrm{a}, \mathrm{b}$ ). The quality of water may be described according to their physico-chemical characteristics (Astel et al., 2006; Shrestha and Kazama, 2007).

For effective maintenance of water quality through appropriate control measures, continuous monitoring of large number of quality parameters is essential. However it is very difficult and laborious task for regular monitoring of all the parameters even if adequate manpower and laboratory facilities are available. Therefore, an attempt based on statistical correlation, has been used to develop mathematical relationship for comparison of physico-chemical parameters.

A number of investigations attempted before to check the water quality assessment with reference to drinking purpose have been carried out in Sagar city (Kowalkowski et al., 2006; Papatheodorou et al., 2006; Barczak and Grivault, 2007; Pathak and Limaye, 2011; 2012; Pathak, 2012; Pathak et al., 2011). 


\section{MATERIALS AND METHODS}

The present work aims to evaluate the bore well water suitability for drinking purpose. 06 sampling places were selected for this study and these are wide spread in the study area. bore well water was collected from July 20018 to Aug. 2019. The water samples were collected in $500 \mathrm{ml}$ polyethylene bottles. All the chemicals used were of AR grade. Analysis was carried out for various water quality parameters such as water temperature measured by using mercury-glass thermometer, $\mathrm{pH}$, conductivity measured by using standard $\mathrm{pH}$ meter and conductivity meter respectively. Total solids (TS) by gravimetric method, total dissolved solids (TDS) by digital conductivity meter, chloride content by argentometric method; Total hardness was calculated by complexometric titration using EDTA titrimetric method, alkalinity by titrimetric method. Dissolved oxygen by Winkler method. by as per Apha (APHA, 2005). The value of the physicochemical parameters were compared with desirable/permissible limit of IS: 10500 drinking water specification (IS-10500:1991). The statistical analysis such as Pearson correlation matrix has been performed using by SPSS 11.0 Statistical Software.

Table 1. Sampling locations and corresponding habitats

Latitude $23^{\circ} 51^{\prime} 16^{\prime \prime} \mathrm{N}$, Longitude 7847'04'E

\begin{tabular}{|l|l|l|}
\hline \multicolumn{1}{|c|}{ Station code-Sampling Locations } & \multicolumn{1}{c|}{ Collection Place } & \multicolumn{1}{c|}{ Sample Source } \\
\hline S1- I. Deendayal Nagar & Residential Area & Bore well water \\
\hline S2-Makronia chouraha & Residential Area & Bore well water \\
\hline S3- Raja khedi & Residential Area & Bore well water \\
\hline S4- Civil line & Residential Area & Bore well water \\
\hline S5-Gopalganj & Residential Area & Bore well water \\
\hline S6- Moti Nagar & Residential Area & Bore well water \\
\hline
\end{tabular}

\section{RESULTS AND DISCUSSION}

The analytical results of physical and chemical parameters of Bore well water were compared with the standard guideline values as recommended by the IS:10500 for drinking and public health purposes. Most Bore well water found in the Sagar city has $\mathrm{pH}$ value ranging from about 7.1 to 8.5 is found to be alkaline in nature. Most of the bore well water samples are within the maximum permissible limit for drinking as per the IS:10500 standard. The value below 510 $\mathrm{mg} / \mathrm{l}$ of TDS, indicating low content of soluble salts in Bore well water water which can be used for drinking without any risk. Site wise estimated values of 10 water quality parameters for 06 Bore well water samples are presented in below tables 2, 3, 4.

Table 2. Water quality physicochemical parameters of different locations of Sagar City at Monsoon (July 2018 to October 2019)

Water Temp. $\left({ }^{\circ} \mathrm{C}\right)$, Colour (Hz.u.), Odour, $\mathrm{pH}$ -

\begin{tabular}{|c|c|c|c|c|c|c|c|c|c|c|}
\hline \multirow{2}{*}{$\begin{array}{c}\text { Station } \\
\text { code }\end{array}$} & \multicolumn{9}{|c|}{ Physico-chemical parameters } \\
\cline { 2 - 12 } & $\begin{array}{c}\text { Water } \\
\text { Temp. }\end{array}$ & $\mathbf{p H}$ & DO & $\begin{array}{c}\text { Conduc } \\
\text { tivity }\end{array}$ & Alkalinity & $\begin{array}{c}\text { Total } \\
\text { Solids }\end{array}$ & TSS & TDS & Chloride & $\begin{array}{c}\text { Total } \\
\text { Hardness }\end{array}$ \\
\hline S1 & 22 & 6.55 & 4.12 & 0.505 & 96 & 316.7 & 8.12 & 308.58 & 45.10 & 230.3 \\
\hline S2 & 24.2 & 7.31 & 4.67 & 0.552 & 141 & 333.68 & 6.35 & 337.23 & 43.21 & 188.56 \\
\hline S3 & 23.5 & 7.53 & 3.05 & 0.534 & 151 & 342.66 & 16.52 & 326.14 & 34.7 & 192.54 \\
\hline S4 & 24.4 & 7.33 & 3.62 & 0.581 & 135 & 378.25 & 22.21 & 356.04 & 30.4 & 186.78 \\
\hline S5 & 24.5 & 6.62 & 5.18 & 0.507 & 152 & 335.35 & 25.25 & 310.10 & 58.8 & 204.85 \\
\hline S6 & 23.5 & 7.43 & 4.63 & 0.674 & 134 & 428.7 & 16.46 & 412.24 & 69.5 & 172.45 \\
\hline
\end{tabular}


Table 3. Water quality physicochemical parameters of different locations of Sagar City at postmonsoon (November 2018 to Feb. 2019)

\begin{tabular}{|c|c|c|c|c|c|c|c|c|c|c|}
\hline \multirow{2}{*}{$\begin{array}{c}\text { Station } \\
\text { code }\end{array}$} & \multicolumn{9}{|c|}{ Physico-chemical parameters } \\
\cline { 2 - 12 } & $\begin{array}{c}\text { Water } \\
\text { Temp. }\end{array}$ & $\mathbf{p H}$ & DO & $\begin{array}{c}\text { Conduc } \\
\text { tivity }\end{array}$ & Alkalinity & $\begin{array}{c}\text { Total } \\
\text { Solids }\end{array}$ & TSS & TDS & Chloride & $\begin{array}{c}\text { Total } \\
\text { Hardness }\end{array}$ \\
\hline S1 & 20.4 & 7.44 & 7.6 & 0.448 & 105 & 303.52 & 5.64 & 297.88 & 34.23 & 223.56 \\
\hline S2 & 19.9 & 8.15 & 8.2 & 0.484 & 158 & 321.54 & 16.52 & 305.02 & 33.26 & 169.76 \\
\hline S3 & 21.0 & 7.87 & 6.7 & 0.483 & 166 & 313.45 & 8.47 & 304.98 & 25.90 & 176.54 \\
\hline S4 & 21.3 & 7.85 & 6.9 & 0.433 & 144 & 330.15 & 9.56 & 320.59 & 26.30 & 172.25 \\
\hline S5 & 22 & 7.83 & 6.7 & 0.471 & 174 & 317.16 & 7.53 & 309.63 & 24.61 & 200.4 \\
\hline S6 & 18.3 & 8.22 & 8.4 & 0.364 & 140 & 338.61 & 17.63 & 320.98 & 35.43 & 163.46 \\
\hline
\end{tabular}

Table 4. Water quality physicochemical parameters of different locations of Sagar City at premonsoon (March 2019 to June 2019)

\begin{tabular}{|c|c|c|c|c|c|c|c|c|c|c|}
\hline \multirow[b]{2}{*}{$\begin{array}{c}\text { Station } \\
\text { code }\end{array}$} & \multicolumn{10}{|c|}{ Physico-chemical parameters } \\
\hline & $\begin{array}{l}\text { Water } \\
\text { Temp. }\end{array}$ & $\mathbf{p H}$ & DO & $\begin{array}{l}\text { Condu } \\
\text { ctivity }\end{array}$ & Alkalinity & $\begin{array}{c}\text { Total } \\
\text { Solids }\end{array}$ & TSS & TDS & Chloride & $\begin{array}{c}\text { Total } \\
\text { Hardness }\end{array}$ \\
\hline S1 & 23.2 & 7.16 & 5.27 & 0.488 & 150 & 424.11 & 17.95 & 406.16 & 48.96 & 242.23 \\
\hline S2 & 25.1 & 7.95 & 5.20 & 0.500 & 146 & 329.68 & 8.56 & 321.12 & 49.97 & 208.52 \\
\hline S3 & 23.4 & 7.82 & 4.35 & 0.499 & 158 & 248.23 & 9.75 & 238.48 & 38.97 & 295.56 \\
\hline S4 & 24.6 & 7.63 & 5.84 & 0.525 & 164 & 315.24 & 9.46 & 305.78 & 40.97 & 201.45 \\
\hline S5 & 24.4 & 7.54 & 4.05 & 0.507 & 151 & 311.93 & 8.47 & 303.46 & 59.97 & 213.46 \\
\hline S6 & 25.3 & 8.0 & 5.15 & 0.526 & 150 & 424.11 & 17.95 & 406.16 & 80.97 & 185.12 \\
\hline
\end{tabular}

It may be suggested that the Bore well water quality of study area can be checked regularly. some prominent correlations exist between water qualities parameters and from correlation values presented in the tables 5, 6, 7 .

Table 5. Matrix of Pearson Correlation for different Parameters in the Borewell waters Samples of in and around Sagar City (Monsoon 2018)

\begin{tabular}{|l|l|l|l|l|l|l|l|l|l|l|}
\hline & P-1 & P-2 & P-3 & P-4 & P-5 & P-6 & P-7 & P-8 & P-9 & P-10 \\
\hline P-1 & 1 & 0.261 & -0.307 & -0.147 & 0.261 & -0.02 & -0.049 & -0.019 & 0.164 & -0.025 \\
\hline P-2 & 0.261 & 1 & 0.37 & 0.62 & 0.412 & 0.691 & 0.763 & 0.002 & 0.638 & 0.123 \\
\hline P-3 & -0.307 & 0.37 & 1 & 0.475 & 0.049 & 0.462 & 0.497 & 0.332 & 0.371 & 0.408 \\
\hline P-4 & -0.147 & 0.62 & 0.475 & 1 & -0.097 & 0.632 & 0.592 & -0.278 & 0.315 & -0.174 \\
\hline P-5 & 0.261 & 0.412 & 0.049 & -0.097 & 1 & 0.402 & 0.359 & 0.217 & 0.588 & 0.293 \\
\hline P-6 & -0.02 & 0.691 & 0.462 & 0.632 & 0.402 & 1 & 0.686 & -0.163 & 0.65 & -0.015 \\
\hline P-7 & -0.049 & 0.763 & 0.497 & 0.592 & 0.359 & 0.686 & 1 & 0.019 & 0.661 & 0.174 \\
\hline P-8 & -0.019 & 0.002 & 0.332 & -0.278 & 0.217 & -0.163 & 0.019 & 1 & -0.143 & 0.979 \\
\hline P-9 & 0.164 & 0.638 & 0.371 & 0.315 & 0.588 & 0.65 & 0.661 & -0.143 & 1 & 0.021 \\
\hline P-10 & -0.025 & 0.123 & 0.408 & -0.174 & 0.293 & -0.015 & 0.174 & 0.979 & 0.021 & 1 \\
\hline
\end{tabular}

Table 6. Matrix of Pearson Correlation for different Parameters in the Borewell waters Samples of in and around Sagar City (PostMonsoon 2018)

\begin{tabular}{|c|c|c|c|c|c|c|c|c|c|c|}
\hline & P-1 & $\mathrm{P}-2$ & P-3 & P-4 & P-5 & P-6 & P-7 & P-8 & P-9 & $\mathrm{P}-10$ \\
\hline $\mathrm{P}-1$ & 1 & 0.267 & 0.188 & 0.537 & 0.266 & 0.934 & 0.836 & 0.935 & 0.491 & 0.916 \\
\hline $\mathrm{P}-2$ & 0.267 & 1 & 0.108 & 0.004 & 0.071 & 0.001 & 0 & 0.994 & 0.002 & 0.605 \\
\hline P-3 & 0.188 & 0.108 & 1 & 0.034 & 0.836 & 0.04 & 0.026 & 0.152 & 0.108 & 0.074 \\
\hline P-4 & 0.537 & 0.004 & 0.034 & 1 & 0.683 & 0.003 & 0.006 & 0.235 & 0.175 & 0.463 \\
\hline
\end{tabular}




\begin{tabular}{|c|c|c|c|c|c|c|c|c|c|c|}
\hline P-5 & 0.266 & 0.071 & 0.836 & 0.683 & 1 & 0.079 & 0.12 & 0.357 & 0.006 & 0.21 \\
\hline P-6 & 0.934 & 0.001 & 0.04 & 0.003 & 0.079 & 1 & 0.001 & 0.492 & 0.002 & 0.951 \\
\hline P-7 & 0.836 & 0 & 0.026 & 0.006 & 0.12 & 0.001 & 1 & 0.938 & 0.002 & 0.463 \\
\hline P-8 & 0.935 & 0.994 & 0.152 & 0.235 & 0.357 & 0.492 & 0.938 & 1 & 0.549 & 0 \\
\hline P-9 & 0.491 & 0.002 & 0.108 & 0.175 & 0.006 & 0.002 & 0.002 & 0.549 & 1 & 0.931 \\
\hline P-10 & 0.916 & 0.605 & 0.074 & 0.463 & 0.21 & 0.951 & 0.463 & 0 & 0.931 & 1 \\
\hline
\end{tabular}

Table 7. Matrix of Pearson Correlation for different Parameters in the Borewell waters Samples of in and around Sagar City (PreMonsoon 2019)

\begin{tabular}{|l|l|l|l|l|l|l|l|l|l|l|}
\hline & P-1 & P-2 & P-3 & P-4 & P-5 & P-6 & P-7 & P-8 & P-10 \\
\hline P-1 & 1 & 0.416 & 0.247 & 0.356 & -0.276 & -0.41 & 0.513 & 0.226 & 0.293 & 0.166 \\
\hline P-2 & 0.416 & 1 & 0.321 & 0.632 & 0.064 & 0.185 & 0.782 & 0.11 & 0.405 & 0.34 \\
\hline P-3 & 0.247 & 0.321 & 1 & 0.366 & 0.225 & 0.179 & 0.176 & -0.521 & 0.257 & -0.357 \\
\hline P-4 & 0.356 & 0.632 & 0.366 & 1 & -0.076 & -0.131 & 0.479 & 0.082 & 0.126 & 0.264 \\
\hline P-5 & -0.276 & 0.064 & 0.225 & -0.076 & 1 & 0.497 & 0.086 & -0.202 & 0.176 & -0.048 \\
\hline P-6 & -0.41 & 0.185 & 0.179 & -0.131 & 0.497 & 1 & 0.051 & -0.262 & 0.45 & -0.036 \\
\hline P-7 & 0.513 & 0.782 & 0.176 & 0.479 & 0.086 & 0.051 & 1 & 0.295 & 0.597 & 0.444 \\
\hline P-8 & 0.226 & 0.11 & -0.521 & 0.082 & -0.202 & -0.262 & 0.295 & 1 & 0.058 & 0.884 \\
\hline P-9 & 0.293 & 0.405 & 0.257 & 0.126 & 0.176 & 0.45 & 0.597 & 0.058 & 1 \\
\hline P-10 & 0.166 & 0.34 & -0.357 & 0.264 & -0.048 & -0.036 & 0.444 & 0.884 & 0.203 & 1 \\
\hline
\end{tabular}

\section{CONCLUSION AND RECOMMENDATIONS}

The major conclusions derived from this study, carried out in the Sagar city are as follows. The physical and chemical parameters of the Sagar citys results shows that all the samples are under recommended limit for drinking purposes. On the basis of detailed chemical analysis, it may be suggested that the regular monitoring must needed for bore well water supply of study area, quality can be checked effectively from the results of the present study, it may be said that, the overall bore well water quality of Sagar is chemically fit for domestic as well as drinking purpose.

\section{REFERENCES}

Astel, A., Biziuk, M., Przyjazny, A., \& Namiesnik, J. (2006). Chemometrics in monitoring spatial and temporal variations in drinking water quality. Water Research, 8, 1706-1716.

Barczak, A., \& Grivault, C. (2007). Système d'Information Géographique pour l'évaluation de la vulnérabilité au risque de ruissellement urbain. NOVATECH 2007.

Herman, G. V., Gaceu, O., Mester, C., Baias, S., Caciora, T., \& Wendt, J. A. (2019a). Spatial Analysis of Water Quality in Natura 2000 Sites Bihor, Romania. Journal of Environmental Protection and Ecology, 20(4), 2121-2129.

Herman, G. V., Ilies, D. C., Gaceu, O., Ilies, A., Mester, C., Ilies, M., \& Dumitru, M. (2019b). Some Considerations Concerning the Quality of Groundwater in the Natura 2000 Lunca Barcaului (Barcaului Meadow) Site, Romania. Journal of Environmental Protection and Ecology, 20(3), 1102-1109.

Kowalkowski, T., Zbytniewski, R., Szpejna, J., \& Buszewski, B. (2006). Application chemometrics in river water classification. Water Research, 40, 744-752.

Papatheodorou, G., Demopoulou, G., \& Lambrakis, N. (2006). A long-term study of temporal hydrochemical data in a shallow lake using multivariate statistical techniques. Ecological Modelling, 193, 759-776.

Pathak, H. (2012). Assessment of physico-chemicalquality of groundwater by multivariateanalysis in somepopulated villages nearby Sagar City, MP, India. J. Environ Anal Toxicol, 2(5), 1-5.

Pathak, H., \& Limaye, S. N. (2012). Assessment of Physico-Chemical Quality of Groundwater in rural area nearby Sagar city, MP, India. Advances in Applied Science Research, 3(1), 555-562.

Pathak, H., \& Limaye, S. N. (2011). Study of seasonal variation in ground water quality of sagar city (India) by principal component analysis. Journal of Chemistry, 8(4), 2000-2009.

Pathak, H., Pathak, D., \& Limaye, S. N. (2011). Seasonal study with interpretation of the chemical characteristics of water pond in reference to quality assessment: A case study. Analele Universitatii din Oradea, Seria Geografie, 21(2), 233-238.

Romocea, T., Oneț, A., Sabău, N.C., Oneț, C., Herman, G.V., \& Pantea, E. (2018). Change of the Groundwater Quality from Industrial Area ORADEA, Romania, Using Geographic Information Systems (GIS). Environmental Engineering \& Management Journal (EEMJ), 17(9), 2189-2199. 
Shrestha, S., \& Kazama, F. (2007). Assessment of surface water quality using multivariate statistical techniques: A case study of the Fuji river basin, Japan. Environmental Modelling and Software, 22, 464-475.

SPSS Advanced Models ${ }^{\text {TM }} 11.0$ Web site at http://www.spss.com

*** (1991). Indian standard drinking water, Specification (First Revision) IS-10500:1991. BIS, New Delhi, India.

*** (2005). APHA, Standard methods for the examination of water and waste water, $21^{\mathrm{st}}$ edition, American Public Health Association, Washington, D.C., USA.

Submitted:

September 27, 2019
Revised:

January 15, 2020
Accepted and published online

March 04, 2020 